\title{
Impact of Quantitative Assessment of Parkinson's Disease-Associated Symptoms Using Wearable Technology on Treatment Decisions
}

We read with interest the report by Santiago et al. [1], demonstrating that clinician decision making regarding the management of motor symptoms of Parkinson's disease (PD) can be enhanced by home-based, continuous objective measurement.

Within the UK National Health Service, we have also been using the Parkinson's Kinetigraph (PKG) since 2015. Similar to the Santiago cohort, in routine care, physicians target PKG use in patients they believe continuous objective measurement will improve the value of clinical encounters. With support from Parkinson's UK, we have carried out an evaluation of utility across seven centers from the Parkinson's Excellence Network, comprising a mix of local services and regional specialist neuroscience centers led by consultant neurologists, geriatricians or Parkinson's nurse specialists. Between July 2015 and July 2017, 256 evaluations were collated, which included information for 209 individual patients relating to PKG impact on clinical decision making. User experience was also assessed by telephone interviews in 155 patients.

Consistent with the findings of Santiago et al. [1], information from the PKG confirmed initial clinical judgement in $54.5 \%$ of cases and provided additional information to inform the clinical decision in $45.5 \%$ cases. Changes in decision making included ten patients where the PKG results prompted a treatment change when clinical assessment alone suggested no changes were necessary, and 15 patients who went on to receive advanced therapies (apomorphine, levodopa-carbidopa intestinal gel or deep brain stimulation) when titration of oral medications was initially considered. Conversely, information from the PKG prompted clinicians to try other options in 18 patients for whom advanced therapies were being considered. Patient feedback was favorable, with $98 \%$ of patients reporting a positive/neutral user experience, most patients finding it simple to use.

Our audit findings replicate those of Santiago et al. [1] in a different healthcare setting, across multiple and varied healthcare provider organizations. The consistency of our evaluation findings highlights the potential of utilizing home-based objective monitoring to facilitate medical management of patients between clinic appointments [2], which holds promise for delivering true home-based care [3].

Camille Carroll (camille.carroll@plymouth.ac.uk), Christopher Kobylecki, Monty Silverdale, Chris Thomas on behalf of the PKG audit group

\section{REFERENCES}

[1] Santiago A, Langston JW, Gandhy R, Dhall R, Brillman S, Rees L, Barlow C (2019) Qualitative evaluation of the Personal KinetiGraphTM Movement Recording System in a Parkinson's clinic. J Parkinsons Dis 9, 207-219.

[2] Hutchinson L, Dominey T, Pearson E, Murphy F, Bell L, Carroll C (2018) Evaluating the clinical utility of the Parkinson's KinetiGraph (PKG) [Abstract]. J Neurol Neurosurg Psychiatry 89(10), 31.

[3] Dorsey ER, Vlaanderen FP, Engelen LJ, Kieburtz K, Zhu W, Biglan KM, Faber MJ, Bloem BR (2016) Moving Parkinson care to the home. Mov Disord 31, 1258-1262. 